\title{
Estudio del efecto de la geometría de un secador solar típico para café con CFD*
}

\author{
Santiago Vélez-Piedrahita* \\ Héctor Ciro-Velásquez ${ }^{* * *}$ \\ Jairo Osorio-Saráz $z^{* * *}$ \\ Esteban Largo-Ávila*****
}

\author{
Recibido: 26/11/2017• Aceptado: 19/06/2018 \\ https://doi.org/10.22395/rium.v18n35a9
}

\begin{abstract}
Resumen
El objetivo de la presente fue modelar el comportamiento térmico de un secador solar típico para café tomando en consideración algunas configuraciones geométricas en la apertura de ventanas, en pos de mejorar las condiciones de secado natural al interior de la estructura. El modelo se simuló con datos experimentales en un secador solar típico en Colombia con Ansys CFX®. Los resultados mostraron que la geometría con una apertura del $25 \%$ en la ventana para la salida del aire aumentó la temperatura sobre la capa de café hasta $4,2{ }^{\circ} \mathrm{C}$ con respecto a la temperatura ambiente, lo cual garantiza uniformidad en el secado, mientras la geometría típica con una apertura del $100 \%$ aumentó la temperatura hasta $3,6^{\circ} \mathrm{C}$, no obstante, con ambas aperturas se aumentó la humedad relativa sobre la capa de café hasta $0,7 \%$ con respecto a la humedad relativa ambiental. Con la apertura máxima de las ventanas disminuyó la humedad relativa del aire.
\end{abstract}

Palabras claves: agroindustria; dinámica de fluido computacional; simulación; energía; temperatura; radiación; secador solar.

Investigación terminada en el marco del proyecto Simulación y validación computacional de un secador solar tipo túnel para café, con tiempo de ejecución de un año, financiado por la Universidad Nacional de Colombia, sede Medellín.

** Ingeniero agrícola, magíster en Ingeniería Agroindustrial. Docente de cátedra, Universidad Nacional de Colombia, sede Medellín y Politécnico Colombiano Jaime Isaza Cadavid, Medellín, Colombia. Correo electrónico: svelezp@ unal.edu.co. Orcid: https://orcid.org/0000-0001-9816-1977

*** Ingeniero mecánico, magíster en Ingeniería Mecánica, doctor en Ingeniería Agrícola. Profesor asociado, Universidad Nacional de Colombia, Medellín, Colombia. Correo electrónico: hjciro@unal.edu.co. Orcid: https:// orcid.org/0000-0002-4398-0470

***** Ingeniero agrícola, magíster en Ingeniería de Materiales y Procesos, doctor en Ingeniería de Alimentos. Profesor asociado, Universidad Nacional de Colombia, Medellín, Colombia. Correo electrónico: aosorio@unal.edu.co. Orcid: https://orcid.org/0000-0002-4358-3600

****** Ingeniero agrícola, magíster en Ciencia y Tecnología de Alimentos. Profesor asistente, Universidad del Valle, sede regional Caicedonia, Colombia. Correo electrónico: esteban.largo@correounivalle.edu.co. Orcid: https:// orcid.org/0000-0001-6047-1523 


\title{
Study of the Effect of the Geometry of a Typical Solar Dryer for Coffee Using CFD
}

\begin{abstract}
The objective of the present investigation was to computationally model a typical solar dryer for coffee, considering some alternative geometric configurations in the opening of windows to improve the thermal conditions within the drying structure which enable the water removal process of wet parchment coffee. The model was simulated in Ansys CFX ${ }^{\circledR}$ software. Simulation results showed that the geometry with an opening of $25 \%$ in the air output window increased temperature in the coffee layer to $4.2{ }^{\circ} \mathrm{C}$ over room temperature, guaranteeing uniform drying, whereas the typical geometry with a $100 \%$ opening increased temperature to $3.8^{\circ} \mathrm{C}$. When the maximum window opening for input and output of air was used, the relative humidity of air within the dryer decreased in comparison with the other geometries.
\end{abstract}

Keywords: agroindustry; computational fluid dynamics; simulation; energy; temperature; radiation; solar dryer.

\section{Estudo do efeito da geometria de um secador solar típico para café com CFD}

\section{Resumo}

O objetivo do presente trabalho foi modelar o comportamento térmico de um secador solar típico para café ao levar em consideração algumas configurações geométricas na abertura de janelas, a fim de melhorar as condições de secagem natural no interior da estrutura. O modelo foi simulado com dados experimentais em um secador solar típico na Colômbia com Ansys CFX ${ }^{\circledR}$. Os resultados mostraram que a geometria com uma abertura de $25 \%$ na janela para saída do ar aumentou a temperatura sobre a camada de café até $4,2{ }^{\circ} \mathrm{C}$ com respeito à temperatura ambiente, o que garante uniformidade na secagem, ao passo que a geometria típica com uma abertura de $100 \%$ aumentou a temperatura até $3,6^{\circ} \mathrm{C}$, não obstante, com ambas as aberturas houve um aumento da umidade relativa sobre a camada de café de até $0,7 \%$ em comparação com a umidade relativa ambiental. Com a abertura máxima das janelas a umidade relativa do ar diminuiu.

Palavras-chave: agroindústria; fluidodinâmica computacional; simulação; energia; temperatura; radiação; secador solar. 


\section{INTRODUCCIÓN}

Uno de los problemas que presentan los caficultores para la comercialización del café pergamino seco es garantizar el contenido de humedad óptimo entre 10 y $12 \%$ base húmeda, lo cual conlleva problemas de calidad y pérdidas al caficultor [1]. Las estructuras de secado solar permiten aumentar la temperatura y reducir la humedad relativa del aire con respecto a las condiciones ambientales, lo cual asegura la viabilidad del dispositivo para el secado de productos [2].

Varios estudios del comportamiento de la temperatura y velocidad del viento en sistemas de secado solar con aplicación en productos agroalimentarios han utilizado dinámica de fluidos computacional (CFD) con resultados numéricos comparables a los datos experimentales [3]. Algunas investigaciones realizaron la optimización de estructuras de secado con modificaciones geométricas [5-6]. Los modelos de elementos finitos se pueden usar para simular el secado de café pergamino y para un diseño óptimo de los secadores de café [7]. Por tanto, la simulación de secadores solares con CFD es útil para el diseño, en pos de garantizar la calidad en el secado y aprovechar al máximo la energía solar, dicha tecnología presenta un uso incipiente en el sector agroalimentario de Colombia.

Con el secador solar en estudio, se presentan tiempos de secado que se pueden prolongar hasta nueve días durante la época de lluvias, comportamiento presente en temporadas con mayores niveles de producción en la zona cafetera colombiana, o que vuelve ineficiente este tipo de sistemas de secado. Por tanto, el objetivo del presente estudio es simular térmicamente un secador solar de café con la herramienta CFD, mediante la modificación de la configuración geométrica en la apertura de ventanas.

\section{MATERIALES Y MÉTODOS}

\subsection{Descripción del secador solar experimental tipo túnel}

El secador solar consta de una estructura en madera, una cubierta plástica y ventanas frontales con cortinas plegables que permiten cambiar la apertura en la entrada y salida del aire. Se encuentra ubicado en el municipio de Fredonia, departamento de Antioquia, localizado en la coordenada $5^{\circ} 52^{\prime} 9,68^{\prime \prime} \mathrm{N} ; 75^{\circ} 40^{\prime} 6,22^{\prime}$ ' O. Las condiciones climaticas medias del sitio corresponden a las presentes en las zonas cafeteras de Colombia con temperatura de $20,2{ }^{\circ} \mathrm{C}$, humedad relativa de $74,0 \%$, y precipitación de $2.674 \mathrm{~mm} /$ año [7]. El secador opera con ventilación natural, tiene un área de secado de $3,85 \mathrm{~m}$ x 9,00 m que permite una capacidad para $409 \mathrm{~kg}$ de café pergamino seco con capa de $3 \mathrm{~cm}$ y una cubierta plástica con forma curva tipo túnel, que permite la incidencia de la radiación solar durante las horas de luz del día, geometría típica en secadores 
utilizados por la caficultura colombiana (figura 1 y figura 2). El plástico utilizado fue polietileno PE calibre 6 con una emisividad de 0,1 .

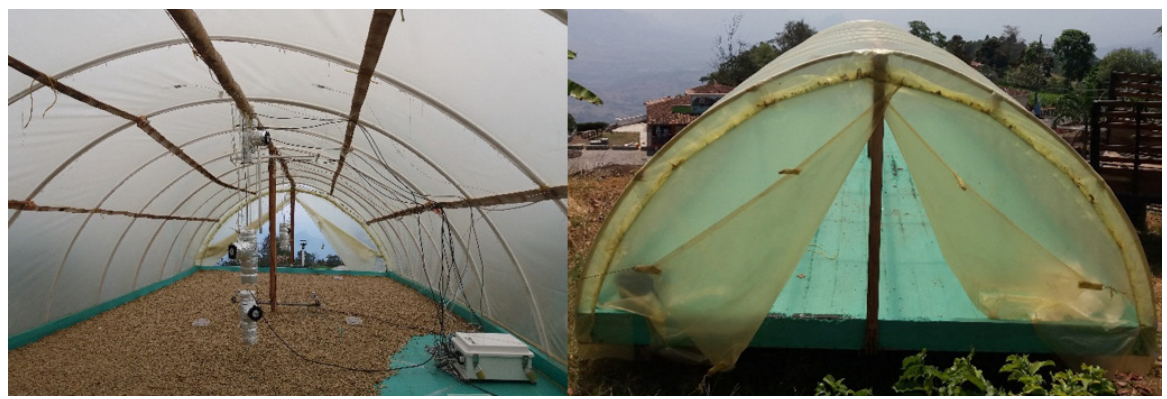

Figura 1. Estructura del secador solar tipo túnel

Fuente: elaboración propia

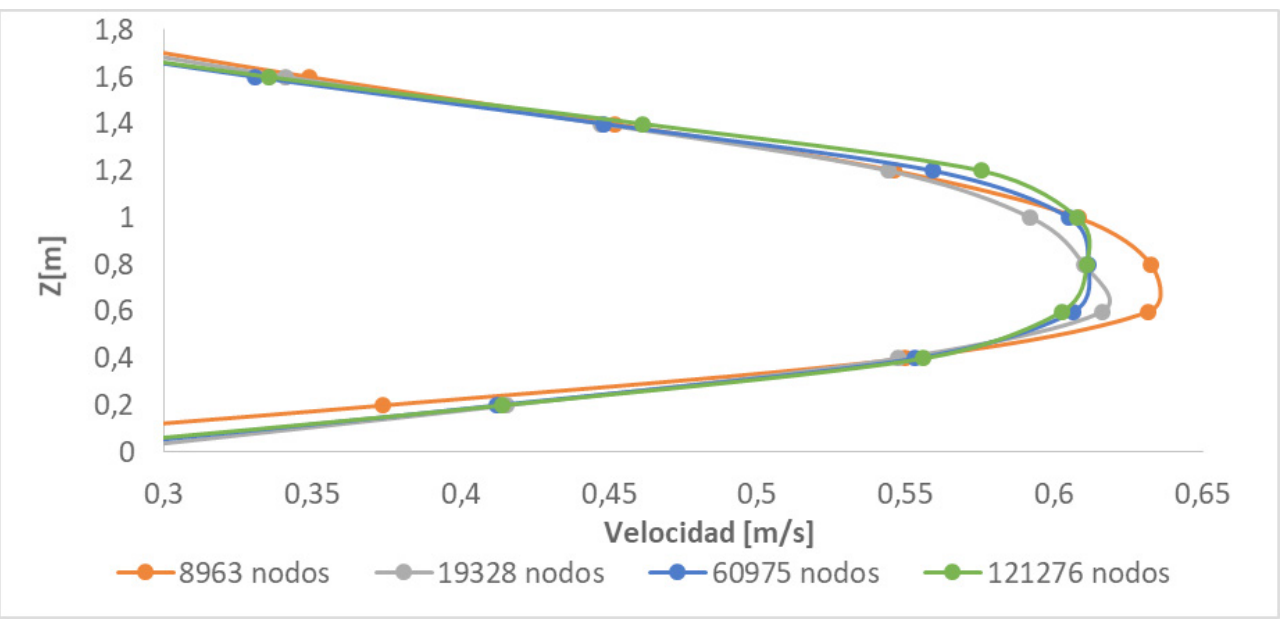

Figura 2. Perfil de la velocidad del viento entre la capa de café y la cubierta plástica, medida en el centro del secador

Fuente: elaboración propia

\subsection{Adquisición de datos experimentales}

La adquisición de datos se realizó con equipos marca Campbell Scientific, mediante la conexión de sensores de temperatura, humedad relativa, velocidad y dirección del viento al datalogger CR1000 [8]. Se instaló un anemómetro WindSonic4 en la ventana a la entrada del aire que permitió medir la velocidad con una resolución de $0,01 \mathrm{~m} / \mathrm{s}$ en un rango de operación entre 0 y $60 \mathrm{~m} / \mathrm{s}$, la dirección del viento se midió con una resolución de $1^{\circ}$. Para la temperatura y humedad relativa del aire en la entrada y salida del aire se utilizó el sensor CS215, para medir temperaturas entre 5 y $40{ }^{\circ} \mathrm{C}$ con una precisión 
de $\pm 0,4{ }^{\circ} \mathrm{C}$ y humedades relativas entre 0 y $100 \%$ con una precisión de $\pm 4 \%$. La temperatura del plástico y del café se midieron con el sensor de temperatura infrarroja IRR-P con precisión de $\pm 0,2{ }^{\circ} \mathrm{C}$ entre -10 y $65^{\circ} \mathrm{C}$. La tasa de agua evaporada desde la capa de café se determinó con el método Gravimet [10], mediante una balanza de precisión modelo PL150E con capacidad maxima para 1.520 g y precisión de $\pm 0,007 \mathrm{~g}$.

Las condiciones climáticas externas al secador solar se midieron con una estación climática marca Decagon Device serie Em50G, ubicada a una distancia de $10 \mathrm{~m}$ y a una altura de $2 \mathrm{~m}$, la medición de temperatura tiene una precisión de $\pm 0,5^{\circ} \mathrm{C}$ entre 5 y $40{ }^{\circ} \mathrm{C}$, para la humedad relativa la precisión es de $\pm 2 \%$ entre 90 y $20 \%$.

\subsection{Dominio computacional}

El modelamiento se realizó con una geometría y malla tridimensional no estructurada, se refinaron nodos en los primeros $10 \mathrm{~cm}$ de las zonas adyacentes a la cubierta plástica y capa de café. Para garantizar calidad en los resultados se realizó una prueba de malla con el programa Ansys ICEM ${ }^{\circledR}$ para evaluar el comportamiento térmico del secador con $8.963,19.328,60.975$ y 121.276 nodos. Los resultados del perfil de velocidad en el centro del secador obtenidos con 60.975 y 121.276 nodos presentaron una tendencia similar, mientras que con 8.963 y 19.328 nodos se presentó mayor dispersión de los resultados (figura 2). No obstante, la malla de 121.276 nodos incrementó considerablemente el tiempo de cómputo, por tanto, se seleccionó la malla generada con 60.975 nodos por presentar la precisión y el costo computacional esperado (figura 3). La computadora utilizada fue una Workstation con procesador Intel Xeon, memoria RAM de 16 GB, disco duro de 1 TB y tarjeta de video Nvidia.

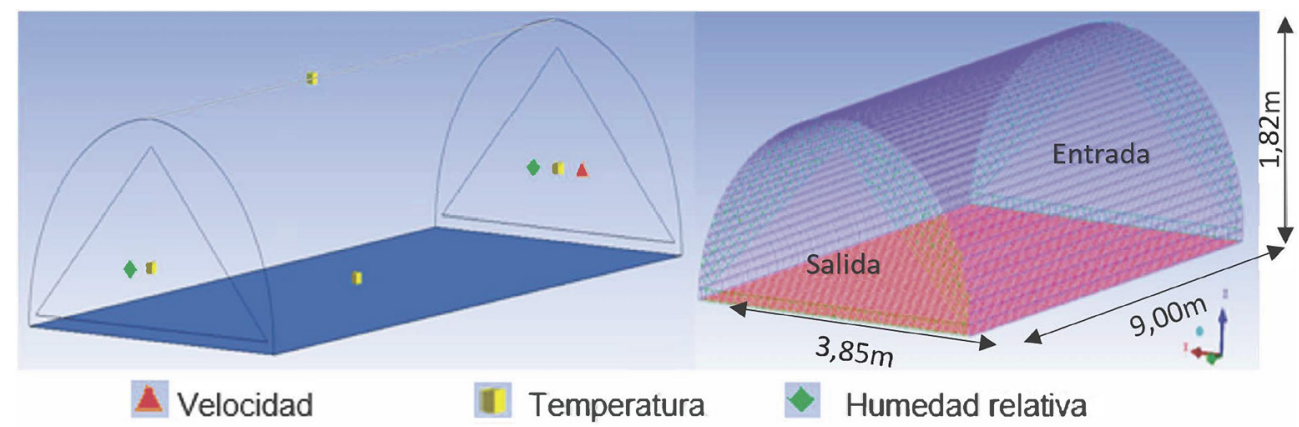

Figura 3. Ubicación de sensores y malla del modelo

Fuente: elaboración propia

El análisis se elaboró con las mediciones experimentales realizadas el 25 de abril del 2016, día en el cual predominaron condiciones climáticas desfavorables en el proceso de secado. En la tabla 1 se registran los valores de entrada constantes obtenidos de manera 
experimental y utilizados en las simulaciones, se operó el secador con la configuración geométrica propuesta T1 (figura 3), correspondiente a la operación habitual del secador. Los valores de temperatura, humedad relativa y velocidad fueron las propiedades del aire en la entrada del secador, mientras el flujo másico fue el agua evaporada desde la capa de café. Todas las propuestas fueron simuladas en estado estacionario a las 2:00 p.m., cuando se presentaron las máximas tasas evaporativas de agua, a las 8:00 a.m. y 6:00 p.m., se realizó la apertura y el cierre de ventanas en la operación habitual del secador, respectivamente.

Tabla 1. Constantes de entrada utilizadas en las simulaciones

\begin{tabular}{|c|c|c|c|c|}
\hline Componente & Temperatura $\left({ }^{\circ} \mathrm{C}\right)$ & $\begin{array}{l}\text { Humedad } \\
\text { relativa (\%) }\end{array}$ & Velocidad $(\mathrm{m} / \mathrm{s})$ & $\begin{array}{l}\text { Flujo másico } \\
\text { de agua }(\mathrm{g} / \mathrm{s})\end{array}$ \\
\hline \multicolumn{5}{|c|}{ 8:00 a. m. } \\
\hline Entrada & 19,8 & 89,7 & 0,42 & \\
\hline Salida & 19,9 & & & \\
\hline Café & 22,6 & & & 0,30 \\
\hline Plástico & 23,7 & & & \\
\hline \multicolumn{5}{|l|}{ 2:00 p. m. } \\
\hline Entrada & 25,2 & 80,8 & 0,62 & \\
\hline Salida & 27,9 & & & \\
\hline Café & 36,9 & & & 1,46 \\
\hline Plástico & 42,7 & & & \\
\hline \multicolumn{5}{|c|}{ 6:00 p. m. } \\
\hline Entrada & 25,9 & 76,9 & 0,18 & \\
\hline Salida & 26,1 & & & \\
\hline Café & 33,4 & & & 0,99 \\
\hline Plástico & 29,9 & & & \\
\hline
\end{tabular}

Fuente: elaboración propia

\subsection{Configuración del modelo computacional}

La simulación se realizó con el paquete comercial Ansys CFX ${ }^{\circledR}$ versión 14 para resolver las ecuaciones de Navier-Stokes que discretizan el dominio del fluido con el método de volúmenes finitos [11]. Las ecuaciones de masa, momentum y energía también se conocen como las ecuaciones de Navier-Stokes y se expresan en términos generales de la forma en la que se presenta en las ecuaciones 1,2 y 3 . 


$$
\begin{gathered}
\frac{\partial \rho}{\partial t}+\nabla \cdot(\rho U)=0 \\
\frac{\partial(\rho U)}{\partial t}+\nabla \cdot(\rho U U)=\nabla p+\mu_{\tau} \nabla U^{2}+\rho g+S_{h} \\
\frac{\partial\left(C_{p} T\right)}{\partial t}+\nabla \cdot\left(-k \nabla T+\rho C_{p} T U\right)=0
\end{gathered}
$$

Donde: $C_{p}$ es el calor específico; [ $\mathrm{J} \mathrm{kg}^{-1}{ }^{\circ} \mathrm{C}^{-1}$ ]. $k$ es la conductividad térmica; [ $\mathrm{W} \mathrm{m}{ }^{-1}$ $\left.{ }^{\circ} \mathrm{C}^{-1}\right]$. $p$ es la presión; $\left[\mathrm{N} \mathrm{m}^{-2}\right]$. $U$ es el vector velocidad; $\left[\mathrm{m} \mathrm{s}^{-1}\right] . \rho$ es la densidad; $\left[\mathrm{kg} \mathrm{m}^{-3}\right]$. $g$ es la gravedad; $\left[\mathrm{m} \mathrm{s}^{-2}\right] . \mu_{\tau}$ es la viscosidad dinámica; $\left[\mathrm{kg} \mathrm{m}^{-1} \mathrm{~s}^{-1}\right]$. $T$ es la temperatura; $\left[{ }^{\circ} \mathrm{C}\right] . S_{h}$ incluye la fuente de calor debido a la incidencia de la radiación solar.

La simulación del secador solar se consideró con el modelo de turbulencia Reynolds Stress BSL por presentar mayor precisión respecto al modelo $k-\varepsilon$. El modelo BSL permite analizar la dinámica de fluidos alrededor de las paredes según el número de Reynolds del aire, al agregar una tensión adicional a la viscosidad dinámica $\mu_{\tau}[11]$.

\subsection{Condiciones de frontera}

Las condiciones de frontera en el modelo CFD se definieron con los siguientes criterios:

1. La humedad relativa se definió como una variable adicional al utilizar las ecuaciones de Tetens 4 y 5 [12].

$$
\begin{gathered}
e_{s}=0,61078 * 10^{\left[\frac{7,5 T}{237,5+T}\right]} \\
e_{v}=\frac{\omega T}{216,68}
\end{gathered}
$$

Donde: $e_{v}$ es la presión de vapor de agua en la atmósfera; $[\mathrm{kPa}] . e_{s}$ es la presión de vapor de agua a saturación; [kPa]. $\omega$ es la humedad absoluta del aire; [ $g$ vapor de agua/kg aire seco]. $T$ es la temperatura del aire, $\left[{ }^{\circ} \mathrm{C}\right]$.

2. La transferencia de calor por radiación se consideró al acoplar el método de Monte Carlo con el término fuente $S_{h}$ de la ecuación de energía de Navier-Stokes [14].

3. La tasa evaporativa de agua desde la capa de café se determinó con el método Gravimet, mediante la ecuación 6.

$$
m_{e v}^{\cdot}=\frac{\Delta m}{\Delta t}
$$


Donde: $m_{e v}$ es la tasa evaporativa de agua en la capa de café $\left.[\mathrm{kg} / \mathrm{s})\right] ; \Delta m$ es el diferencia de masa de café humedo [kg]; $\Delta t$ es el intervalo de tiempo en la medición de la masa de café humedo [s].

4. La entrada de aire a través de la ventana y la tasa evaporativa de agua en la capa de café se consideraron uniformes espacialmente.

\subsection{Propuestas geométricas en el secador solar}

El tiempo de secado depende del diseño geométrico del secador solar [14]. La optimización de un secador para frutas mediante modificaciones geométricas con CFD permitió aumentar la temperatura del aire y lograr una distribución uniforme del flujo [5].

La figura 4 muestra las seis propuestas con diferentes configuraciones geométricas en la apertura de ventanas con ventilación natural que se evaluaron en el presente estudio. La geometría T1 corresponde a la configuración original del secador con ventanas en la entrada y salida del aire de igual dimensión y apertura del $100 \%$, las geometrías T2, T3 y T4 solo tienen modificaciones en la ventana a la salida del aire con aperturas triangulares del $55 \%, 40 \%$ y $25 \%$ con relación a la apertura original de la geometría T1, respectivamente. La geometría T5 tiene la salida del aire a traves de una sección rectangular en la parte inferior, y la geometría T6 corresponde a la apertura máxima de ambas ventanas, al suprimir el material plástico de los costados frontales.

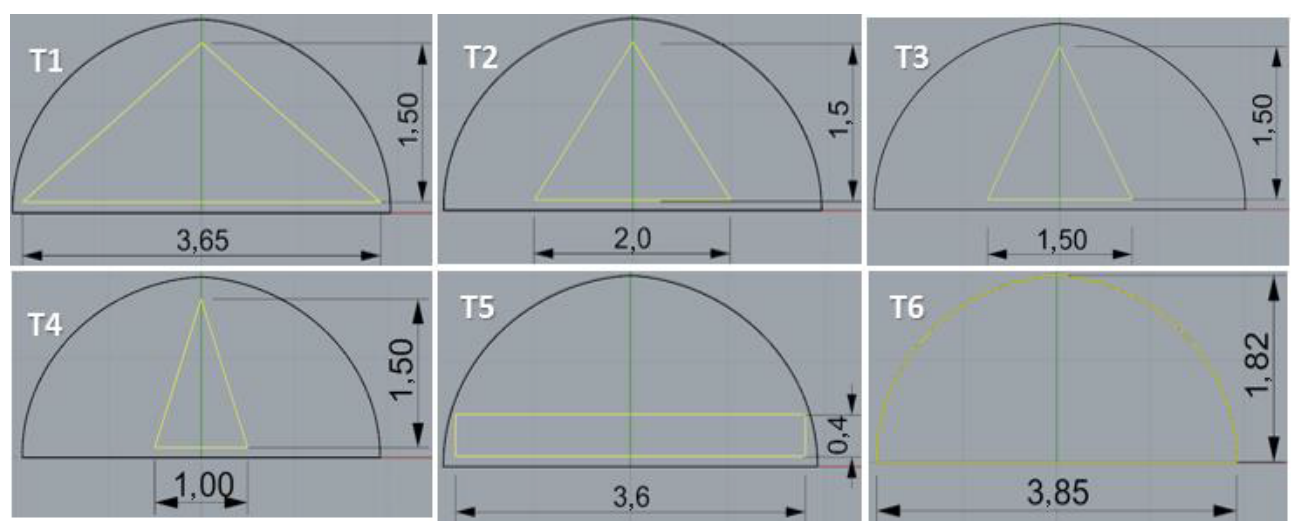

Figura 4. Dimensiones de la ventana a la salida del aire para las configuraciones geométricas propuestas (unidades en metros)

Fuente: elaboración propia 


\subsection{Análisis estadístico}

Para analizar estadísticamente las diferencias entre las configuraciones geométricas propuestas respecto a la temperatura y la humedad relativa en un plano a $10 \mathrm{~cm}$ sobre la capa de café, se realizó una prueba de comparación de medias con una prueba T-student con un nivel de significancia del $5 \%$.

\section{RESULTADOS Y DISCUSIÓN}

\subsection{Comportamiento térmico en el interior y exterior del secador con operación original T1}

La viabilidad del secador solar se determina mediante la diferencia de temperatura y de humedad relativa con respecto a las condiciones ambientales [2]. Los datos experimentales medidos en el interior y exterior del secador solar, con la configuración geométrica T1, evidencian que la temperatura del aire en el interior fue superior a la temperatura ambiental: la diferencia de temperatura fue $1,9^{\circ} \mathrm{C}, 3,6$ ${ }^{\circ} \mathrm{C}$ y $2,5{ }^{\circ} \mathrm{C}$ a las 8:00 a.m., 2:00 p.m., y 6:00 p.m., respectivamente, mientras que la humedad relativa disminuyó $0,5 \%, 10,4 \%$ y $6,8 \%$ a las 8:00 a.m., 2:00 p.m., y 6:00 p.m. Janjai et al. [16], obtuvieron un incremento de temperatura de $8{ }^{\circ} \mathrm{C}$ aproximadamente, en un secador solar tipo túnel con ventilación forzada ubicado en un ambiente tropical.

\subsection{Análisis de temperatura y humedad relativa entre configuraciones geométricas}

El análisis de temperatura y humedad relativa en las seis configuraciones geométricas propuestas se realizaron con los resultados de las simulaciones en un plano a $10 \mathrm{~cm}$ sobre la capa de café $Z=10 \mathrm{~cm}$, al considerar que la tasa de evaporación de agua, el tiempo de secado y la calidad en taza dependen de las condiciones térmicas del aire adyacente al grano, este hecho ha sido ampliamente estudiado en secado mecánico [16].

En la tabla 2 se indica que hay diferencias significativas $(\mathrm{p}<0,05)$ en la temperatura y humedad relativa de la geometría T6 durante las horas objeto del estudio, lo cual evidencia que la máxima apertura de ventanas no generó aumento de la temperatura, y una disminución de la humedad relativa útil para el proceso de secado. No obstante, se presenta una diferencia significativa en la temperatura de la geometría $\mathrm{T} 4$ a las 2:00 p.m., y 6:00 p.m., que puede aumentar entre $3,6{ }^{\circ} \mathrm{C}$ y $4,2{ }^{\circ} \mathrm{C}$ en el plano $\mathrm{Z}=$ $10 \mathrm{~cm}$ con respecto a la temperatura ambiental. El análisis anterior logra predecir mayores temperaturas cuando la ventana a la salida del aire tiene una apertura del 25 $\%$ (geometría T4), mientras la humedad relativa no presentó un aumento que afecte el proceso, lo cual permite aumentar la tasa de evaporación y disminuir el tiempo de secado del café [2]. 
Tabla 2. Valores medios e intervalos de confianza para la temperatura y humedad relativa en el plano $Z=10 \mathrm{~cm}$ sobre la capa de secado a las 8:00 a.m., 2:00 p.m., y 6:00 p.m.

\begin{tabular}{|c|c|c|c|c|c|c|c|}
\hline \multirow[b]{2}{*}{ Geometría } & \multirow[b]{2}{*}{$N$} & \multicolumn{2}{|c|}{8 a.m. } & \multicolumn{2}{|c|}{2 p.m. } & \multicolumn{2}{|c|}{$6 p . m}$. \\
\hline & & $\begin{array}{c}\text { Temperatura } \\
\left({ }^{\circ} \mathrm{C}\right)\end{array}$ & $\begin{array}{l}\text { Humedad } \\
\text { relativa (\%) }\end{array}$ & $\begin{array}{c}\text { Temperatura } \\
\left({ }^{\circ} \mathrm{C}\right)\end{array}$ & $\begin{array}{l}\text { Humedad } \\
\text { relativa (\%) }\end{array}$ & $\begin{array}{c}\text { Temperatura } \\
\left({ }^{\circ} \mathrm{C}\right)\end{array}$ & $\begin{array}{l}\text { Humedad } \\
\text { relativa (\%) }\end{array}$ \\
\hline $\mathrm{T} 1$ & 27 & $20,3 \pm 0,29^{a}$ & $89,81 \pm 0,04^{a}$ & $27,1 \pm 1,21^{\mathrm{a}}$ & $81,1 \pm 0,15^{\mathrm{a}}$ & $27,0 \pm 0,62^{\mathrm{a}}$ & $77,5 \pm 0,31^{\mathrm{a}}$ \\
\hline $\mathrm{T} 2$ & 27 & $20,3 \pm 0,35^{\mathrm{a}}$ & $89,80 \pm 0,04^{\mathrm{a}}$ & $27,3 \pm 1,40^{\mathrm{a}}$ & $81,1 \pm 0,15^{\mathrm{a}}$ & $27,0 \pm 0,66^{\mathrm{a}}$ & $77,5 \pm 0,31^{\mathrm{a}}$ \\
\hline $\mathrm{T} 3$ & 27 & $20,3 \pm 0,33^{\mathrm{a}}$ & $89,80 \pm 0,05^{\mathrm{a}}$ & $27,5 \pm 1,38^{\mathrm{a}}$ & $81,1 \pm 0,15^{\mathrm{a}}$ & $27,2 \pm 0,65^{\mathrm{a}}$ & $77,5 \pm 0,31^{\mathrm{a}}$ \\
\hline $\mathrm{T} 4$ & 27 & $20,3 \pm 0,34^{\mathrm{a}}$ & $89,80 \pm 0,05^{\mathrm{a}}$ & $28,0 \pm 1,39^{\mathrm{c}}$ & $81,1 \pm 0,16^{\mathbf{a}}$ & $27,5 \pm 0,65^{\mathrm{c}}$ & $77,5 \pm 0,31^{\mathrm{a}}$ \\
\hline T5 & 27 & $20,2 \pm 0,26^{\mathrm{a}}$ & $89,80 \pm 0,04^{\mathrm{a}}$ & $27,0 \pm 1,09^{\mathrm{a}}$ & $81,1 \pm 0,14^{a}$ & $26,9 \pm 0,58^{\mathrm{a}}$ & $77,5 \pm 0,29^{a}$ \\
\hline T6 & 27 & $19,9 \pm 0,10^{\mathbf{b}}$ & $89,77 \pm 0,04^{\mathrm{b}}$ & $25,4 \pm 0,34^{\mathbf{b}}$ & $80,9 \pm 0,11^{\mathbf{b}}$ & $26,2 \pm 0,29^{b}$ & $77,2 \pm 0,24^{\mathbf{b}}$ \\
\hline
\end{tabular}

Nota: valores con la misma letra significa que no existe diferencia significativa $(\mathrm{p}<0.05)$

Fuente: elaboración propia

Un análisis estadístico previo de la pérdida de peso indicó un secado uniforme de la capa de café con la geometría T1, lo cual evidencia que la distribución de las condiciones térmicas al interior del secado solar no genera diferencias significativas en la pérdida de peso entre diferentes posiciones con la geometría T1. Por consiguiente, al comparar la desviación estándar de la geometría T1 con las demás geometrías registradas en la Tabla 2, se deduce que el comportamiento térmico de las geometrías propuestas en los tratamientos no afecta negativamente la uniformidad del secado, toda vez que las desviaciones estándar con la geometría T1 son similares a las desviaciones de las geometrías T2, T3, T4, T5 y T6, y fueron calculadas con datos de temperatura y humedad relativa obtenidos en el proceso de simulación en 27 posiciones uniformemente distribuidas sobre el plano $\mathrm{Z}=10 \mathrm{~cm}$.

En las figuras 5, 6 y 7 se observa la distribución espacial de temperatura y humedad relativa en el plano $Z=10 \mathrm{~cm}$ a las 8:00 a.m., 2:00 p.m., y 6:00 p.m., respectivamente. En todas las geometrías e instantes de tiempo se da un aumento de temperatura durante la circulación del aire desde la entrada hacia la salida, la humedad relativa presenta un comportamiento similar producto de la evaporación de agua desde la capa de café. 


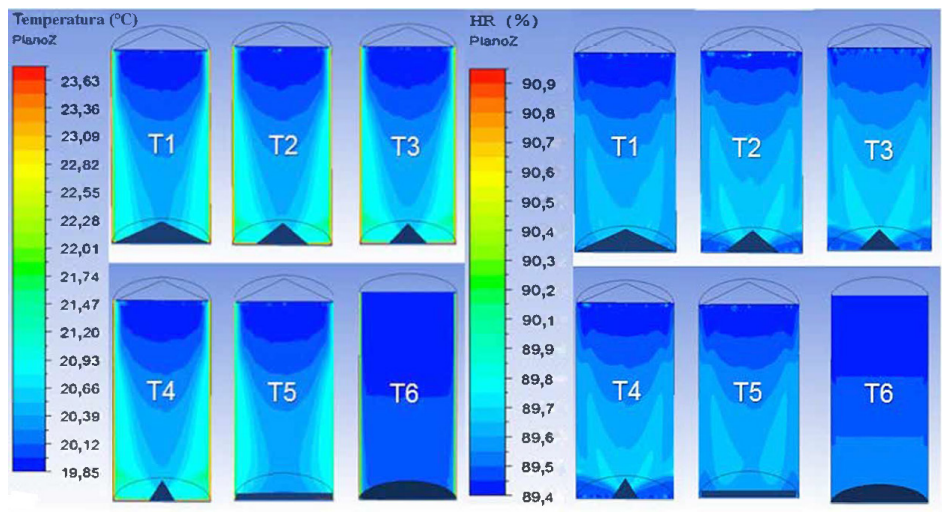

Figura 5. Comportamiento de la temperatura y humedad relativa en el plano $Z=10 \mathrm{~cm}$ a las 8:00 a.m. Fuente: elaboración propia

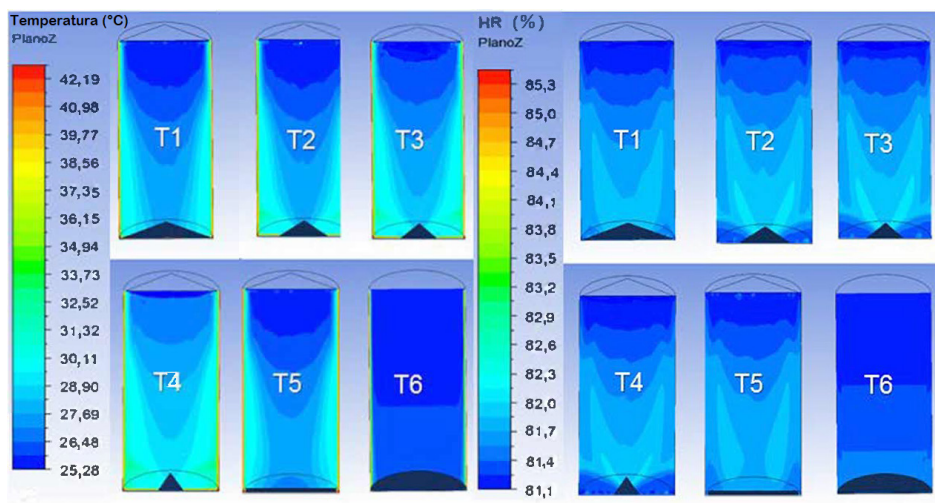

Figura 6. Comportamiento de la temperatura y humedad relativa en el plano $Z=10 \mathrm{~cm}$ a las 2:00 p.m. Fuente: elaboración propia

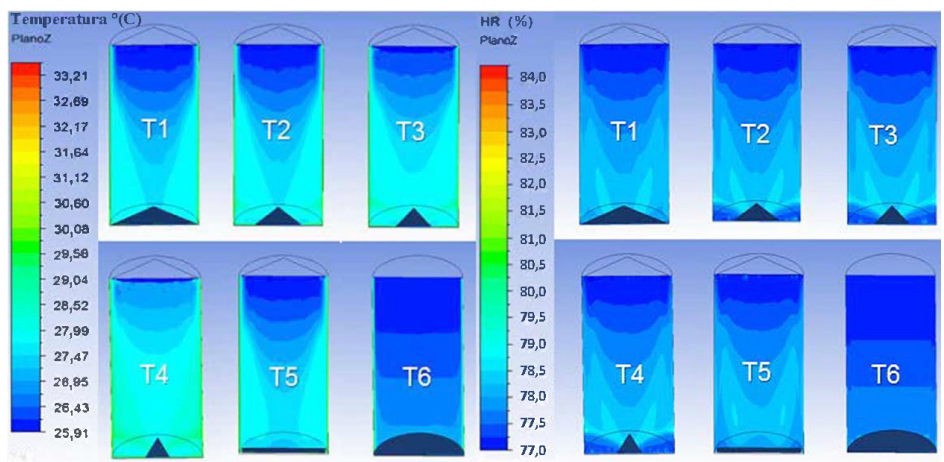

Figura 7. Comportamiento de la temperatura y humedad relativa en el plano $Z=10 \mathrm{~cm}$ a las $6: 00$ p.m. Fuente: elaboración propia 
La temperatura de la capa de café y del plástico fueron superiores a la temperatura del aire que ingresa al secador solar, lo que generó los mayores diferenciales térmicos en las zonas adyacentes a dichos materiales, producto de la transferencia de calor por convección.

La geometría T6 presenta mayor uniformidad de la temperatura y humedad relativa por tener apertura en todo el ancho de la ventana a la salida del aire, no obstante, el incremento de temperatura es mínima con respecto a la temperatura atmosférica por la alta tasa de ventilación interna o renovación del aire generada con la apertura total.

En los instantes de tiempo simulados, las máximas temperaturas en el plano Z=10 $\mathrm{cm}$ se presentaron en la geometría T4; la apertura del $25 \%$ de la ventana en la salida del aire restringe la transferencia de calor hacia el exterior del secador solar, además, la radiación en onda larga o infrarroja reflejada entre la capa de café y el plástico calienta el aire interno e intensifica el efecto invernadero [14].

\section{CONCLUSIONES}

La dinámica de fluidos computacional CFD fue una herramienta versátil y confiable para la comparación térmica en diferentes configuraciones geométricas para la apertura de ventanas del secador solar.

Los resultados de la simulación muestran que la geometría T4 con apertura del $25 \%$ en la ventana a la salida del aire fue adecuada para aumentar la temperatura de manera uniforme sobre la capa de café; no se recomienda implementar la geometría T6 con la apertura máxima de ambas ventanas en el secado solar por el poco incremento de temperatura, hasta de $0,3{ }^{\circ} \mathrm{C}$ en épocas lluviosas.

La apertura máxima de las ventanas en la salida y entrada del aire correspondiente a la geometría T6 permitió disminuir hasta $0,7 \%$ la humedad relativa del aire con respecto a las demás geometrías, producto de la alta tasa de renovación del aire.

\section{AGRADECIMIENTOS}

Los autores agradecen a la Universidad Nacional de Colombia, sede Medellín, por proporcionar los equipos utilizados en el proyecto de investigación. Además, un reconocimiento a la administración de la finca Santa Isabel del municipio de Fredonia, departamento de Antioquia, por ofrecer el secador solar tipo túnel objeto de estudio.

\section{REFERENCIAS}

[1] B. Soediono, "Beneficio del café II: secado del cafe pergamino," J. Chem. Inf. Model., vol. 53, p. 160, 1989. 
[2] A. G. Ferreira, C. B. Maia, M. F. B. Cortez, and R. M. Valle, "Technical feasibility assessment of a solar chimney for food drying," Sol. Energy, vol. 82, n. ${ }^{\circ}$ 3, pp. 198-205, 2008.

[3] C. Brasil, A. Ferreira, L. Cabezas, S. Morais y T. Oliveira Martins, "Simulation of the airflow inside a hybrid dryer," Arpapress, vol. 10, n. ${ }^{\circ}$ 3, pp. 382-389, 2012.

[4] Y. Amanlou and A. Zomorodian, "Applying CFD for designing a new fruit cabinet dryer," J. Food Eng., vol. 101, no. 1, pp. 8-15, 2010.

[5] R. Alvarez and J. Santamaria, "Dinámica de fluidos computacional aplicada al estudio de regeneradores térmicos," Dyna, vol. 71, no. March, pp. 81-93, 2004.

[6] W. Nilnont, S. Thepa, S. Janjai, N. Kasayapanand, C. Thamrongmas, and B. K. Bala, "Finite element simulation for coffee (Coffea arabica) drying," Food Bioprod. Process., vol. 90, n. 2 , pp. 341-350, 2012.

[7] Ideam, “Clima,” Ideam, [En línea], Disponible: http://www.ideam.gov.co/web/tiempo-y-clima/ clima?inheritRedirect=true, 2010

[8] I. Campbell Scientific, "CR1000 Measurement and Control System," Campbell Scientific, Logan, Utah, pp. 1-678. Disponible: https://shop.profec-ventus.com/images/Datasheets/ Data_loggers/SCI/CR1000/cr1000-manual-2015.pdf, 2015.

[9] J. M. Jurado-Chaná, E. C. Montoya-Restrepo, C. E. Oliveros-Tascón, and J. García-Alzate, "Método para medir el contenido de humedad del café pergamino en el secado solar del café," vol. 60, n. ${ }^{\circ} 2$, pp. 135-147, 2009.

[10] J. Osorio, I. Ferreira, K. Olivera, L. Barreto, and T. Norton, "a Cfd Based Approach for Determination of Ammonia Concentration Profile and Flux From Poultry Houses With Natural Ventilation," Rev. Fac. Nac. Agron., vol. 69, n. . 1, pp. 7825-7834, 2016.

[11] Ansys, Ansys CFX-Solver Theory Guide, Canonsburg, Pensilvania: Ansys, 2013.

[12] C. B. Alvarenga et al., "Effect of the water vapor pressure deficit in the air on hydropneumatic spraying of artificial targets," Biosci. J., vol. 30, n. ${ }^{\circ}$ 1, pp. 182-193, 2014.

[13] P. Verboven, A. K. Datta, N. T. Anh, N. Scheerlinck, and B. M. Nicolaï, "Computation of airflow effects on heat and mass transfer in a microwave oven," J. Food Eng., vol. 59, n. . 2-3, pp. 181-190, 2003.

[14] J. Besora, "Informe Técnico para la Construcción de un Secador Solar de Café," Ing. Sin Front., 2016.

[15] S. Janjai, P. Intawee, J. Kaewkiew, C. Sritus, and V. Khamvongsa, "A large-scale solar greenhouse dryer using polycarbonate cover: Modeling and testing in a tropical environment of Lao People's Democratic Republic," Renew. Energy, vol. 36, n. 3, pp. 1053-1062, 2011.

[16] J. Henao, "Evaluación del proceso de secado del café y su relación con las propiedades físicas, composición química y calidad en taza," Tesis, Universidad Nacional de Colombia, sede Medellín, 2016. 